\title{
Treatment challenges for alcohol service users in Kampala, Uganda
}

\author{
David Kalema $^{1,2}$, Wouter Vanderplasschen ${ }^{1}$, Sofie Vindevogel ${ }^{3}$, Peter K. Baguma ${ }^{2}$, and Ilse Derluyn ${ }^{4}$ \\ ${ }^{1}$ Department of Special Needs Education, Faculty of Psychology and Educational Sciences, Ghent University, Ghent, Belgium \\ ${ }^{2}$ School of Psychology, College of Humanities and Social Sciences, Makerere University, Kampala, Uganda \\ ${ }^{3}$ Department of Orthopedagogics, Faculty of Education, Health and Social Work, University College Ghent, Ghent, Belgium \\ ${ }^{4}$ Department of Social Work and Social Pedagogy, Faculty of Psychology and Educational Sciences, Ghent University, Ghent, Belgium
}

\begin{abstract}
Background and Aims: Enhancing treatment participation of persons with substance use disorders is a challenge worldwide. Obstacles keeping people from entering or continuing treatment are well documented in Western countries, but such knowledge is scarce in majority countries that face particular challenges when implementing alcohol policies. This study aimed at identifying factors challenging treatment participation in Uganda, a Sub-Saharan country with a considerable alcohol problem.
\end{abstract}

Methods: Data were collected during 30 in-depth, qualitative interviews on treatment challenges with 20 service providers and 10 male service users, who were recruited at one public and one private alcohol treatment center in the Ugandan capital city, Kampala. Men comprise about $90 \%$ of the total number of service users in these centers. Interview data were analyzed thematically, using Nvivo software, and were categorized around three levels of treatment challenges: societal, institutional, and personal challenges.

Findings: Interview findings showed several treatment challenges relating to institutional aspects like inadequate human resources, overall insufficiency of services, and the treatment philosophy of available services. Respondents identified stigma and cultural interference as important challenges at the societal level, while limited awareness about addiction and denial of problems can be situated at the individual level.

Conclusions: Institutional, societal, and personal challenges keep persons with AUD from participating in alcohol treatment in public and private services in Uganda. Alcohol regulation, sensitization, and prevention are needed to raise awareness at the societal and individual level, while appropriate training and additional financial resources may help to overcome institutional challenges.

\section{Introduction}

Alcohol use disorders (AUD) are a growing public health concern in Sub-Saharan Africa. In Uganda, high per capita alcohol consumption, heavy episodic drinking, and underage alcohol use are well documented (Swahn, Palmier, \& Kasirye, 2013; World Health Organization [WHO], 2014). Based on a recent international comparison (Graham et al., 2011), the negative personal and social consequences among drinkers were estimated to be among the highest globally and call for effective interventions (WHO, 2010). Literature on alcohol treatment in Uganda is scarce (Kalema, Vindevogel, Baguma, Derluyn, \& Vanderplasschen, 2015) but indicates that the majority of problem users fail to access professional treatment (Kullgren, Alibusa, \& Birabwa-Oketcho, 2009; Ministry of Health $[\mathrm{MoH}], 2010)$. Although low treatment access and participation is recognized as a problem worldwide (Rehm, Shield, Rehm, Gmel, \& Frick, 2012), some of the challenges may be specific to developing countries due to contextual differences like political and economic instability, the influence of the alcohol industry, and the role of religion in daily life (Al-Ansari, Thow, Day, \& Conigrave, 2015; Kalema, Vanderplasschen, Vindevogel, \& Derluyn, 2016). Consequently, identification of obstacles that interfere with service and treatment utilization in these countries may help to improve treatment participation and quality of service provision.

Based on the WHO STEPwise approach to surveillance of non-communicable diseases, the proportion of Ugandans with an AUD was estimated at 9.8\% (3,920,000 people) in 2014 (Kabwama et al., 2016). Hospital-based studies indicate higher prevalence rates among HIV/AIDS patients (47\%) and persons entering primary health care centers (17\%) (Hahn et al., 2014; Kullgren et al., 2009). According to the WHO (2010), treatment is an essential recommended intervention for reducing AUD, but it is relatively new and rare in Uganda and scantly documented (Ndyanabangi, 2013). Mainstream health care facilities are the official first contact points for persons with AUD, but

Correspondence: David Kalema, Ghent University, Department of Special Needs Education, Henri Dunantlaan, 2, B-9000 Ghent, Belgium. Telephone: +256-772 481 003; E-mail: kalemdav@yahoo.com

Keywords: alcohol abuse, addiction, treatment barriers, Sub-Saharan Africa, Uganda, qualitative research 
these services do not provide more than detoxification (MoH, 2010). Spiritual, traditional, and other forms of informal healing are significant features of mental health care in Sub-Saharan Africa, but remain largely unknown to the scientific community (Odejide, Oheri, Adelekan, \& Ikuesan, 1992).

Uganda has one specialized public AUD treatment facility at the National Referral Hospital in Kampala and eight private, small-scale non-governmental organizations around the capital city that offer specialized residential treatment lasting 90 days. Treatment in these facilities is based on the traditional Minnesota model (Kalema \& Vanderplasschen, 2015), which regards chemical addiction as a chronic and progressive disease and emphasizes abstinence from all mood-altering substances to achieve recovery. It consists of a combination of the Alcoholics Anonymous 12-step program and pharmacological and psychosocial interventions (National Institute on Drug Abuse [NIDA], 2000). Some authors (Klingemann \& Bergmark, 2006) have questioned the appropriateness of the Minnesota model as a primary treatment solution for AUD in resource-limited settings. This is likely to be compounded by stigma, as the model is based on selfrevelation (Salwan \& Katz, 2014). Moreover, it is unclear whether late adjustments to address the limitations of the Minnesota model (NIDA, 2000) have been adopted in Uganda. Also, aftercare following residential treatment is only provided occasionally.

According to the Ugandan Bureau of Statistics (UBOS), $49 \%$ of Uganda's population is younger than 15 years of age (UBOS, 2014) and can hence be regarded as the second youngest and one of the fastest-growing populations worldwide. This exceptional demographic situation, in combination with a high burden of disease and low per capita income (World Bank, 2015), limits the prioritization of mental health care in the public sector (Kigozi et al., 2010). Private services are expensive and hence unaffordable for the general population. Access to treatment is further hampered by the fact that alcohol services are concentrated in urban areas around Kampala (Ndyanabangi, 2013), while the majority of people with AUD live in rural areas (MoH, 2014). Paradoxically, service providers are suspicious of spiritual treatment and other types of informal support (Barker \& Hunt, 2007), although these culturally based initiatives are easily accessible, trusted by the general population, and cheaper than institutionalized health care facilities (Odejide et al., 1992). Consequently, large shortages are experienced in alcohol service delivery, and available resources are subject to severe pressure. For example, in the only public psychiatric hospital, only two beds and one staff member are available per 100,000 inhabitants (Kigozi et al., 2010). Further, available facilities suffer from culturally and/or religiously induced stigma, which reduce acceptability of these services (Massachusetts General Hospital, 2015). Moreover, although English is considered the official national language, 43 different languages are currently in use in Uganda, which can make communication challenging, in particular with less educated and illiterate persons (22\% of the population) (Ethnologue.com, 2017).
Some recent publications (Ndyanabangi, Basangwa, Lutaakome, \& Mubiru, 2009; Kigozi et al., 2010) have revealed challenges and prospects for mental health care in Uganda and called for research on the functioning of mental health services to guide future reforms.

Factors that have been associated with poor treatment access and participation among persons with AUD and other substance use disorders in the international literature include personal challenges (e.g., negative social support, unaffordability, privacy and time concerns, shame, stigma, and denial), as well as system challenges (e.g., lack of expertise, limited availability, intake procedures and requirements) (Fin, Bakshi, \& Andréasson, 2014; Myers, Louw, \& Fakier, 2008; Myers, Petersen, Kader, \& Parry, 2012; Pasche, Kleintjes, Wilson, Stein, \& Myers, 2015; Rapp et al., 2006). Identified obstacles appear to be similar across countries and cultures, although their magnitude may differ according to place and population. For example, in a U.S. study Schuler, Oheri, Adelekan, and Ikuesan (2015) highlighted attitudinal aspects (i.e., readiness to change) as a main obstacle to treatment in general, while Browne and colleagues (2016) reported primarily service-related challenges in rural areas (e.g., transportation costs, absence of technology). Although research on this issue is rare in Africa, Myers et al. (2012) mentioned awareness of treatment options, geographic access, and affordability as major facilitators to alcohol and other drug treatment among Black Africans and Colored South Africans. Societal stigma is a recurring barrier in most treatment access studies, which has a significant impact on the acceptability of services and motivation for treatment (Ebigbo, Elekwachi, \& Nweze, 2012; Keyes et al., 2010; Saunders, Zygowicz, \& D'Angelo, 2006).

Research on alcohol-related disorders in Uganda is limited and treatment issues have barely been studied (Kalema \& Vanderplasschen, 2015). Also, most studies focus on public facilities, while $46 \%$ of all health services in Uganda are provided by private, non-governmental organizations (U.S. Agency for International Development [USAID], 2005). Since alcoholism is considered a unique disorder requiring a specialized approach (Sterling, Chi, \& Hinman, 2000), we attempt to fill the knowledge gap on alcohol treatment in Uganda by studying treatment challenges for persons with AUD as perceived by service users and providers in private and public services. By doing so, these findings may help to improve the accessibility and quality of alcohol services in Uganda and other Sub-Saharan countries.

\section{Methods}

To explore facilitating and impeding factors associated with AUD treatment in Uganda, we used a qualitative study design. Qualitative research was used because this method makes it possible to explore individuals' diverse experiences with treatment and their subjective perspectives of these experiences (Lucassen \& Olde Harteman, 2007). 
Study sample: A total of 30 participants was chosen purposefully from two AUD treatment facilities in Kampala, Uganda. One site was selected because it is the only public specialized facility, while the other was a typical private center (small-scale and non-profit, but in which clients have to pay for services). The public facility is a 25-bed unit/ward at the National Referral Hospital, while the private center has a bed capacity of 15 clients. Both facilities offer similar programs (as explained in the introduction) and will be referred to as site 1 and site 2 respectively, while presenting the results.

In both centers, we interviewed 10 service providers and 5 service users in the period of October-November 2014. The administrators of both programs selected respondents, based on the eligibility criteria. Service providers were key therapeutic staff and an administrator, all directly charged with treatment of AUD clients. Eligible service users were those diagnosed with alcohol dependence, in residential treatment for at least two months, and with a stable psychological and physical state at the time of the interview. We included service users because they are the primary beneficiaries of treatment and their experiences are crucial in evaluating alcohol services. A greater number of providers was selected to represent the multidisciplinary composition of the treatment staff. Also, respondents had to speak English.

The average age of interviewed treatment providers was 35 years; 13 were male and 7 were female. Their professional backgrounds varied: 13 were allied health professionals (2 psychologists, 8 counselors, and 3 social workers), 2 psychiatric nurses, 2 general nurses, 2 psychiatric clinical officers, and 1 psychiatrist. They had been working in AUD treatment for, on average, 5 years. All service users were men, and their mean age was 29 years. Half of them were students in higher education (four at university and one in high school), and the other five respondents were university graduates who reported that they were unemployed prior to admission. Although the sample is too small to represent characteristics of males in treatment, the overrepresentation of highly educated alcohol users depicts an affluent group that can afford treatment. Also, the high number of school-going respondents may not be a surprise, given the young average age of the population (UBOS, 2014) and the high prevalence of teenage drinking (Kafuko \& Bukuluki, 2008; Swahn, Haberlen, \& Palmier, 2014). Service users reported to have been drinking for 13 years on average, and all were in treatment for the first time. Only male respondents were selected for this study, since the number of female service users is very low (around
1:10) and none was eligible/willing to participate at the time of study selection.

Data collection: Data were collected using in-depth interviews consisting of open-ended questions that lasted 45 to 90 minutes and were administered at the respective treatment facilities. Interviews focused on difficulties experienced by alcohol users in seeking and staying in treatment, their level of satisfaction with the services offered, causes of drop-out and relapse, and the general population's attitude towards alcohol users and services (see Table 1). No incentives were provided for study participation. The study was approved by the Ugandan National Council of Science and Technology and by the ethical boards of the two supervising universities. To ensure independence of the interviewer, interviews were conducted by a Belgian final-year master's student in special needs education. Informed consent was obtained from all study participants, and with their permission, interviews were recorded with an audio device. Although the interviews were conducted in English, respondents were allowed to express themselves in their local language whenever necessary.

Data Analysis: Interviews were transcribed verbatim. Collected data were structured using thematic analysis (Braun \& Clarke, 2006) and analyzed with Nvivo 11 computer software. While analyzing the interviews, emerging themes were clustered around three types of factors: societal, institutional, and personal factors (see Figure 1). The three levels were created inductively and emerged as categories of challenges, while the nodes should be regarded as themes and subtypes. To strengthen the reliability and validity of the study findings, the results were discussed by some of the authors and staff members from research site 2 .

\section{Results}

Responses revealed closely interconnected facilitating and impeding factors, ranging from negative societal attitudes to institutional/service-based limitations and personal challenges (see Figure 1). Selected quotes are used to illustrate each theme. As shown in Table 1, responses on societal factors were clustered around following themes: awareness, cultural aspects, and availability of alcohol (see Table 1). Themes at the institutional level included human resources, quality of services, treatment options, intake capacity, and costs. Low motivation (non-acceptability) was coded under individual characteristics. 


\section{Table 1}

Overview of interview questions and themes and subthemes emerging among interviewees

\begin{tabular}{|c|c|c|}
\hline Interview question & Category (Challenge) & Nodes and Sub-nodes \\
\hline $\begin{array}{l}\text { To Service Providers (Only) } \\
\text { - Why do most people with alcohol use } \\
\text { - } \quad \text { Wroblems fail to seek treatment? } \\
\text { - What are the major causes of client drop- } \\
\text { - What causes relapse among clients? } \\
\text { - What is the biggest challenge to alcohol }\end{array}$ & Societal & $\begin{array}{l}\text { Low awareness levels (of disease and services); } \\
\text { Misconception/myths about alcohol/alcohol } \\
\text { abuse, alcohol abuse treatment, and users of } \\
\text { alcohol abuse treatment } \\
\text { Cultures and religion; As a challenge to recovery } \\
\text { in and out of treatment } \\
\text { Availability of alcohol }\end{array}$ \\
\hline $\begin{array}{l}\text { To Both Service Providers and Users } \\
\text { - What is the general view of people on: } \\
\text { - Alcohol abuse treatment and } \\
\text { - Users of alcohol treatment programs? }\end{array}$ & Institutional & $\begin{array}{l}\text { Human Resources; } \\
\text { Adequacy (work load), Remuneration/motivation, } \\
\text { and Expertise/skills } \\
\text { Quality of services; User satisfaction, } \\
\text { Appropriateness of therapeutic techniques and } \\
\text { materials }\end{array}$ \\
\hline $\begin{array}{l}\text { To Service Users (Only) } \\
\text { - } \quad \text { Please explain if it was difficult for you to } \\
\text { find treatment } \\
\text { - } \quad \text { Does the treatment environment and } \\
\text { programme meet your expectations? }\end{array}$ & & $\begin{array}{l}\text { Treatment options; Approaches, facilities and } \\
\text { alternatives } \\
\text { Limited intake capacity } \\
\text { Costs; Affordability }\end{array}$ \\
\hline $\begin{array}{l}\text { Please explain. } \\
\text { What are the major challenges to your } \\
\text { recovery? }\end{array}$ & Individual & $\begin{array}{l}\text { Characteristics; } \\
\text { Low motivation (Non-acceptability) }\end{array}$ \\
\hline
\end{tabular}

Figure 1

Identified clusters of treatment challenges among interviewees

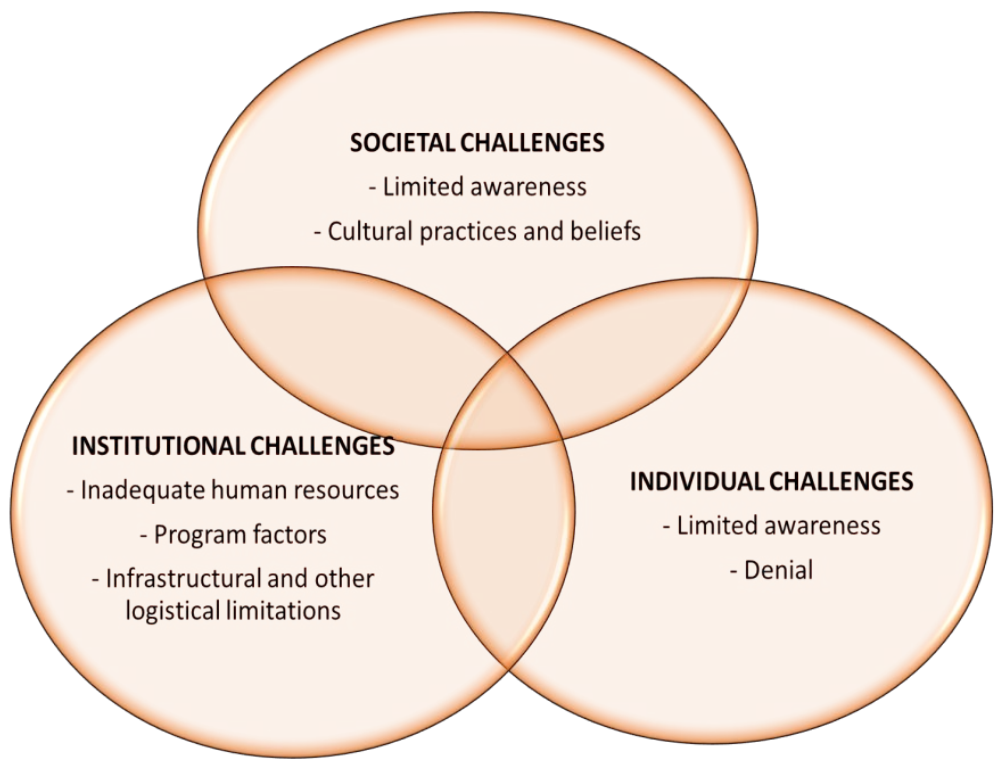

\section{Societal Challenges}

Limited awareness: Numerous respondents cited exposure to alcohol at young age and widespread public drunkenness as common societal features of alcohol abuse in Uganda, but awareness among the general population is restricted. Limited awareness can be regarded a societal as well as an individual challenge. Several respondents stated that many persons with AUD do not know that alcoholism is a treatable disorder, nor where to access services: "Those who know it [treatment] think that it doesn't work at all. Most of them mock us. They think, ah, we are wasting time . . . and money," stated a service user at site 2. Service users and providers at both sites reported several myths that still 
prevail in the population, such as "You can't be an addict and successful in life" or "Treatment is for those who are badly off." The view that "alcoholism is suffered by those who are mentally ill" is also common and is compounded by the fact that public AUD treatment is provided in mental health care units. Yet others think that alcoholism is curable with herbs or medicines and see no need for professional treatment. Societal lack of trust in treatment is aggravated by the perceived high relapse rates after treatment.

Cultural practices and beliefs: Respondents blamed the dominant culture for the wide availability and acceptability of alcohol, which in itself promotes heavy drinking and relapse among (treated) individuals. Some ethnic groups believe that "alcoholism is bewitched" and insinuatealong with some religious groups-that "alcohol abuse is a curse and requires exorcism instead of treatment." On top of condoning alcohol, intercultural issues may interfere with treatment, as service providers reported communication difficulties with non-English speaking users from different tribes.

\section{Institutional/service-based challenges}

Inadequate human resources: Service providers reported repeatedly about the heavy workload, which is a serious threat to the quality of service delivery. To address this problem, volunteers and interns are recruited as additional workforce, which may raise concerns regarding their competence and motivation.

"If . . . they want to go, they go. . . . After we trained people, they disappeared. The student psychologists are temporal, so if it is not yet time for that course, then you are not going to see them. That is why you are going to find that we are seasonal. There are seasons when it is good and when it is really bad" (psychologist, site 1 ).

Also, some respondents admitted that staff members lack essential knowledge for the management of AUD. "We actually don't understand what addiction is. Sometimes, you can even ask some doctors these things and they don't know" (social worker, site 1).

Program factors: Service users, especially from site 1, complained about congestions in the therapeutic sessions and monotony of the topics presented. Service providers attributed this to staff shortage: "At times we resort to a 'one shoe [size] fits all'-approach. I don't want it as well," said a psychologist at site 1 . The program leanness is compounded by lack of therapeutic materials such as literature and other visual/learning tools at both sites, hence limiting the ability of users to facilitate their own recovery processes. Moreover, insufficient recreational and therapeutic options are reported at both sites, as illustrated by this statement of a clinical psychiatric officer at site 2: "No Antabuse [is available], we are just on detoxification. For other [treatment] models, we only stop at reading about them, but we cannot practice because we have to go by what is available. For example, we lack an occupational center." Finally, the treatment program lasts 90 days, but this period is considered too long by service users as it affects their work, study, and family commitments. "My studies ... [are] something . . crucial in my life, so when someone tells me: 'get a dead year, first work on yourself, and then go back to school,' it was a setback," stressed a user at site 1 .

Infrastructural and other logistic limitations: The bed capacity at site 1 is reportedly low compared to the demand. Hence, many users have to be on a waiting list long before admission. Although offering paid services, private treatment is as well limited in capacity and cannot absorb all persons who cannot be treated in public facilities. One counselor (site 2) explained: "When you look at the resources, accommodation, and even the meals, it's too expensive for these people. There is no support by the government." To create space, treatment norms and expectations are moderated, and in most cases all types of clients are treated together, including persons with severe psychiatric disorders. This challenge was mentioned more frequently at site 1 but was also acknowledged at site 2 . A counselor from site 2 stressed some negative consequences of this lack of differentiation: "When some are psychotic . . . they are aggressive, resentful, and are in denial. They become violent and vandalize property, it becomes a very stressing environment for both the clients and staff."

\section{Individual challenges}

As mentioned above, limited awareness is a societal as well as an individual challenge. Societal stigma reinforces denial and resistance towards residential treatment among persons with AUD. Not surprisingly, the majority of admissions are involuntary, those who have been tricked into treatment or only agreed to treatment after extensive persuasion.
"Clients also have a challenge, because it is not their motivation. Many times, those are the people you talk to and they always try to fake the recovery-'You see, I am now okay, I sleep, I understand the problem, now; when am I going?'”' (psychologist, site 1)

A counselor at site 2 reported that the majority of users are resentful of the program and fail to cooperate. Also, some residents try to sneak alcohol or other drugs in the treatment center and eventually escape, which demotivates other service users and frustrates the staff.

\section{Discussion}

Based on 30 qualitative interviews with service providers and users at two treatment centers in the capital city of Kampala, we identified perceived challenges for persons with AUD in Uganda related to individual, institutional, and societal aspects of seeking and receiving treatment. As noted earlier in this paper, some challenges occur at more than one level (Rapp et al., 2006; Schuler et al., 2015). According to the interviewees, Uganda's case is characterized by positive societal attitudes toward alcohol and negative beliefs about treatment, limited human and 
infrastructural resources, poor quality of service delivery, and individual resistance to treatment. It is noteworthy that the service user sample consisted of highly educated individuals, illustrating that treatment is primarily accessible to affluent citizens (Schuler et al., 2015).

The wide availability of alcohol in Ugandan society and its consideration as an ordinary drink (Swahn et al., 2013; WHO, 2010) were repeatedly mentioned by service providers and users as major societal challenges to treatment access and retention. Over the past two decades, Sub-Saharan Africa has witnessed a rapid increase in the availability and affordability of alcohol, primarily as a result of the alcohol industry's attempts to open new markets for their products (Jernigan \& Obot, 2006; Willis, 2006; Kalema et al., 2016). This specific context, combined with ignorance about alcohol addiction and its treatment, as well as persistent societal challenges such as poverty, infectious diseases (e.g., HIV), and internal conflicts, prevents people not only from seeking treatment but also from staying in treatment. In the absence of effective alcohol regulations, prevention, and sensitization initiatives (Kalema \& Vanderplasschen, 2015), problem awareness is limited.

Addressing alcohol problems is further complicated by cultural and traditional beliefs. Research participants indicated that formal alcohol treatment services and spiritual and traditional healers appear to be two distinct support systems that can be considered opposing rather than cooperating factors. The conceptualization of alcoholism as an "incurable disease" by the predominant Minnesota model is an institutional approach which appears to be inconsistent with the local culture, as numerous people believe that persistent illness is due to improper treatment or evil influence. Some spiritual testimonies have claimed to provide total healing from alcoholism (Odejide et al., 1992), which may explain the popularity of traditional and spiritual healers. Evaluation studies are needed as well regarding the effectiveness of Minnesota-based and other formal treatment modalities compared to traditional and spiritual healers.

Most challenges that were cited by service providers and users concerned agency functioning and availability. While the only public alcohol treatment clinic is overwhelmed by the high demand from the population, private initiatives lack adequate resources to avail their services to all persons who are unable to access inexpensive public services. This finding is consistent with previous studies in the SubSaharan region that showed a great shortage of public mental health care facilities (Myers et al., 2012). The interviews further revealed that service provision is compromised by the sector's inability to attract and retain skilled staff, as has been found for the mental health sector in Uganda (Kigozi et al., 2010).

Ugandan service providers identified denial as a major individual barrier to treatment access, while other authors (Schuler et al., 2015) have also pointed at psychiatric comorbidity, insufficient financial resources, and stigma as main barriers. Denial and resistance are regarded as inherent aspects of addiction and behavioral change processes (e.g., Cunningham, Blomqvist, \& Cordingley, 2007; Rinn, Desai, Rosenblatt, \& Gastfriend, 2002), but low motivation and poor treatment adherence are equally typical for other chronic disorders (e.g., diabetes, hypertension) (McLellan, 2002). Consequently, making treatment more attractive to potential users and providing motivational, low threshold, and continuing interventions are important challenges for alcohol services in Uganda.

Low treatment utilization among women is a particular feature of Ugandan alcohol treatment, which was illustrated by the lack of female participants in this study and a 10:1 male-to-female ratio in alcohol services overall. In other African countries, this low representation has been attributed to cultural taboo and stigma, particularly among women who are unemployed, impoverished, and/or uneducated (Ebigbo et al., 2012; Myers, 2011). Moreover, as illustrated by the high educational status of most study participants, services are generally unable to attract people with low educational attainment and low income, despite $24 \%$ of the Ugandan population living below the poverty line (Ebigbo et al., 2012; Population Secretariat \& United Nations Population Fund [UNFPA], 2012).

Given the multidimensional nature of the identified challenges to treatment participation and the fact that most respondents associated the perceived failure of treatment services to the increased availability of alcohol, insufficient human and infrastructural resources, and the poor quality of services, measures at the societal and institutional level are needed. Comprehensive alcohol policy regulations (Babor et al., 2010) and adequate sensitization and prevention strategies (Kalema \& Vanderplasschen, 2015) are likely to increase awareness at the population level. Moreover, such measures may contribute to the development of an intervention spectrum from primary prevention to longterm treatment (Mrazek \& Haggerty, 1994).

Second, considering the role played by both private and public services in alcohol treatment in Uganda (USAID, 2005), better collaboration between both sectors could increase available resources and enhance systematic planning of AUD interventions at various levels (Center for Substance Abuse Treatment, 2000; Pedersen et. al., 2015). For example, this could be done by pooling staff and resources, by providing common training and supervision sessions, or by shared decision making. Public-private collaboration was also a key recommendation by the Ugandan Population Secretariat \& UNFPA (2012), since such partnerships enhance service delivery and complement each other in situations of scarce resources. Collaboration and networking are also emphasized in Uganda's Mental Health Care Plan as key ingredients for more effective mental health services (Ndyanabangi, 2013).

Third, since research participants from both facilities complained about limited capacity of available services, additional and affordable treatment slots need to be made available. Also, the predominant medical-psychiatric approach should be complemented with more comprehensive approaches and community-based practices 
that support persons with AUD in their natural environments. Challenges regarding staff expertise and competence were described by respondents and could be improved by recruiting more allied health workers (e.g., social workers, psychologists) in specialized services and by providing specialist training to primary health care workers (e.g., counseling and support skills, motivational interventions) (WHO, 2010).

Finally, to remove societal barriers and address the limited English language proficiency for uneducated or minimally educated alcohol users, educational and therapeutic programs in local languages could increase awareness and reduce stigma and denial among individuals and communities.

\section{Limitations}

As research on alcohol treatment in Uganda is still in its infancy, several limitations should be noted. First, the study sample was limited to two residential treatment centers, excluding other services or stakeholders that apply different treatment philosophies from the Minnesota model. Also, since only 10 (male) service users were interviewed, the range of perspectives and experiences is small. For example, our sample of affluent and highly educated individuals is likely to leave out opinions of service users with lower/no educational attainment. It is recommended to include a more diverse sample in future research, including females and service users from lower socioeconomic status groups. Moreover, involving persons who dropped out of treatment or who were awaiting treatment and/or did not seek treatment at all would provide complementary perspectives regarding treatment challenges. Finally, all respondents were selected by the administrators of both programs, which may have led to a selection bias as they could choose to select individuals who were most eloquent or those likely to report positively about treatment and service provision in Uganda. However, all interviews were administered in a separate room and confidentiality was assured to all respondents.

\section{Conclusion}

Challenges and difficulties in the delivery of alcohol treatment in Uganda are attributed by service providers and service users to a combination of societal, institutional, and individual factors. Reforms at various levels are necessary to provide more effective treatment services and to attract and engage a wider range of persons with AUD. In particular, the quality of service delivery should be improved through training and additional human and infrastructural resources. Also, alcohol treatment should be extended to include outpatient and outreach activities, while alcohol regulation, education, and prevention programs are needed to increase awareness about addiction and reduce related stigmatization.

\section{References}

Al-Ansari, B., Thow, A., Day, C., \& Conigrave, K. (2015). Extent of alcohol prohibition in civil policy in Muslim majority countries: The impact of globalization. Addiction, 111(10):1703-1713. doi:10.1111/ add.13159

Babor, T., Caetano, R., Casswell, S., Edwards, G., Giesbrecht, N., Graham K., . . . Rossow, I. (2010). Alcohol: No ordinary commodity (2nd ed.). Oxford, England: Oxford University Press.

Barker, J. C., \& Hunt, G. (2007). Natural recovery: A cross-cultural perspective. In J. C Barker \& G. Hunt (Eds.), Promoting self-change from addictive behaviours (pp. 213-237). New York, NY, United States: Springer US.

Braun, V., \& Clarke, V. (2006). Using thematic analysis in psychology. Qualitative Research in Psychology, 3, 77-101.

Browne, T., Priester, M. A., Clone, S., Iachini, A., DeHart, D., \& Hock, R. (2016). Barriers and facilitators to substance use treatment in the rural south: A qualitative study. Journal of Rural Health, 32(1), 92101. doi:10.1111/jrh.12129

Center for Substance Abuse Treatment. (2000). Integrating substance abuse treatment and vocational services (Treatment Improvement Protocol Series, Number 38). Rockville, MD, United States: Substance Abuse and Mental Health Services Administration.

Cunningham, J. A., Blomqvist, J., \& Cordingley, J. (2007). Beliefs about drinking problems: Results from a general population telephone survey. Addictive Behaviors, 32, 167-169.

Ebigbo, P. O., Elekwachi, C. L., \& Nweze, C. F. (2012). On the cutting edge of modern developments in psychotherapy. Journal of Contemporary Psychotherapy, 42(4), 257-264. doi:10.1007/s10879012-9213-9

Ethnologue.com. (2017). Ethnologue languages of the world: Uganda. Retrieved from www.ethnologue.com

Finn, W. S., Bakshi, A. S., \& Andreasson, S. (2014). Alcohol consumption, dependence, and treatment barriers: perceptions among nontreatment seekers with alcohol dependence. Substance Use \& Misuse, 49(6), 762-769. doi:10.3109/10826084.2014.891616

Graham, K., Bernards, S., Knibbe, R., Kairouz, S., Kuntsche, S., Wilsnack, S. C., . . . Gmel, G. (2011). Alcohol-related negative consequences among drinkers around the world. Addiction, 106, 1391-1405.

Hahn, J., Fatch, R., Wanyenze, R. K., Baveewo, S., Kamya, M. R., Bangsberg, D. R., \& Coates, T. J. (2014). Decreases in self-reported alcohol consumption following HIV counseling and testing at Mulago Hospital, Kampala, Uganda. BMC Infectious Diseases, 14, 403.

Jernigan, D. H., \& Obot, I. (2006). Thirsting for the African market. African Journal of Drug \& Alcohol Studies, 5, 57-70.

Kabwama, S. N., Ndyanabangi, S., Mutungi, G., Wesonga, R., Bahendeka, S. K., \& Guwatudde, D. (2016). Alcohol use among adults in Uganda: Findings from the countrywide non-communicable diseases risk factor cross-sectional survey. Global Health Action, 9. doi:10.3402/gha.v9.31302

Kafuko, A., \& Bukuluki, B. (2008). Qualitative research in Uganda on knowledge, attitudes and practices 
concerning alcohol. Retrieved from https://www.k4health.org/sites/default/files/Alcohol\% 20Study\%20Report\%20FINAL\%20March\%2013th.pd $\mathrm{f}$

Kalema, D., \& Vanderplasschen, W. (2015). Features and challenges of alcohol abuse treatment in Uganda. African Journal of Drug \& Alcohol Studies, 14(2), 125-135.

Kalema, D., Vanderplasschen, W., Vindevogel, S., \& Derluyn, I. (2016). The role of religion in alcohol consumption and demand reduction in Muslim majority countries (MMC). Addiction, 111(10), 17161718. doi:10.1111/add.13333

Kalema, D., Vindevogel, S., Baguma, P., Derluyn, I., \& Vanderplasschen, W. (2015). Alcohol misuse, policy and treatment responses in Sub-Saharan Africa: The case of Uganda. Drugs: Education, Prevention and Policy, 22(6), 476-482.

Keyes, K. M., Hatzenbuehler, M. L., McLaughlin, K. A., Link, B., Olfson, M., Grant, B. F., \& Hasin, D. (2010). Stigma and treatment for alcohol disorders in the United States. American Journal of Epidemiology, 172(12), 1364-1372.

Kigozi, F., Ssebunnya, J., Kizza, D., Cooper, S., Ndyanabangi, S., \& The Mental Health and Poverty Project. (2010). An overview of Uganda's mental health care system: Results from an assessment using the World Health Organization's assessment instrument for mental health systems (WHOAIMS). International Journal of Mental Health Systems, 4(1), 1-9. Retrieved from http://www.ijmhs.com/content/ $4 / 1 / 1$

Klingemann, H., \& Bergmark, A. (2006). The legitimacy of addiction treatment in a world of smart people. Addiction, 101(9), 1230-1237.

Kullgren, G., Alibusa, S., \& Birabwa-Oketcho, H. (2009). Problem drinking among patients attending primary healthcare units in Kampala, Uganda. African Journal of Psychiatry, 12, 52-58.

Lucassen, J., \& Olde Hartman, C. (2007). Kwalitatief onderzoek: Praktische methoden voor de medische praktijk (Translation: Qualitative research: Practiceoriented methods for the medical practice). Houten, Nederland: Bohn Stafleu van Loghum.

Massachusetts General Hospital. (2015). Combating barriers to care in rural Uganda. Retrieved from http://www.mghcgh.org/stories-from-thefield/combating-barriers-to-care-in-rural-uganda/

McLellan, A.T. (2002). Have we evaluated addiction treatment correctly? Implications from a chronic care perspective. Addiction, 97, 249-252.

Ministry of Health. (2010). Uganda clinical guidelines. Kampala, Uganda: Government of Uganda.

Ministry of Health. (2014). Non-Communicable Disease Risk Factor Baseline Survey. Uganda 2014 report. Kampala, Uganda: Government of Uganda.

Mrazek, P. J., \& Haggerty, R. J. (1994). Reducing the risks for mental disorders: Frontiers for preventive intervention research. Washington, DC, United States: National Academy Press.

Myers, B. (2011). Barriers to alcohol and other drug treatment use among Black African and Coloured
South Africans. African Journal of Psychiatry, 14, 146-153.

Myers, B., Louw, J., \& Fakier, N. (2008). Alcohol and drug abuse: Removing structural barriers to treatment for historically disadvantaged communities in Cape Town. International Journal of Social Welfare, 17(2), 156-165.

Myers, B., Petersen, Z., Kader, R., \& Parry, C. D. H. (2012). Moving beyond access: Towards a qualityorientated substance abuse treatment system in South Africa. South African Medical Journal, 102(8), 667668.

National Institute on Drug Abuse. (2000). Minnesota model: Description of counseling approach. In P. Owen (Ed.), Approaches to drug abuse counseling (pp. 117-126). Washington, DC, United States: National Institute of Health.

Ndyanabangi, S., Basangwa, D., Lutaakome, J., \& Mubiru, C. (2009). Uganda mental health country profile. International Review of Psychiatry, 16(1-2), 54-62. Retrieved from http://www.tandfonline.com/doi/abs/ 10.1080/09540260310001635104

Odejide, A. O., Oheri, J. U., Adelekan, M., \& Ikuesan, B. A. (1992). Alcohol-treatment issues in Nigeria: Traditional healers and modern psychiatry. In $\mathrm{H}$. Klingemann, J. Takala, \& G. Hunt (Eds.), Cure, care or control - Alcoholism treatment in 16 countries (pp. 277-284). Albany, NY, United States: State University of New York Press.

Pasche, S., Kleintjes, S., Wilson, D., Stein, D. J. \& Myers, B. (2015). Improving addiction care in South Africa: Development and challenges to implementing training in addictions care at the University of Cape Town. International Journal of Mental Health and Addiction, 13(3), 322-332.

Pedersen, E. R., Eberhart, N. K., Williams, K. M., Tanielian, T., Epley, C., \& Scharf, D. M. (2015). Public-private partnerships for providing behavioral health care to veterans and their families: What do we know, what do we need to learn, and what do we need to do? Santa Monica, CA, United States: RAND Corporation. Retrieved from www.ncbi.nlm.nih.gov/ pubmed/28083394

Population Secretariat \& United Nations Population Fund. (2012). The state of Uganda population report 2012: Uganda at 50 years: Population and service delivery; Challenges, opportunities and prospects. Kampala, Uganda: Population Secretariat \& United Nations Population Fund.

Rapp, R. C., Xu, J., Carr, C. A., Lane, D. T., Wang, J., \& Carlson, R. (2006). Treatment barriers identified by substance abusers assessed at a centralized intake unit. Journal of Substance Abuse Treatment, 30(3), 227235.

Rehm, J., Shield, K. D., Rehm, M. X., Gmel, G., \& Frick, U. (2012). Alcohol consumption, alcohol dependence and attributable burden of disease in Europe: Potential gains from effective interventions for alcohol dependence. Toronto, Canada: Centre for Addiction and Mental Health.

Rinn, W., Desai, N., Rosenblatt, H., \& Gastfriend, D. R. (2002). Addiction denial and cognitive dysfunction. 
Journal of Neuropsychiatry and Clinical Neurosciences, 14(1), 52-57.

Salwan, J., \& Katz, C. L. (2014). A review of substance use disorder treatment in developing world communities. Annals of Global Health, 80(2), 115-121.

Saunders, S. M., Zygowicz, K. M., \& D'Angelo, B. R. (2006). Person-related and treatment-related barriers to alcohol treatment. Journal of Substance Abuse Treatment, 30(3), 261-270. doi: 10.1016/j.jsat.2006. 01.003

Schuler, M. S., Puttaiah, S., Mojtabai, R., \& Crum, R. M. (2015). Perceived barriers to treatment for alcohol problems: A latent class analysis. Psychiatric Services, 66(11), 1221-1228.

Sterling, S., Chi, F., \& Hinman, A. (2000). Integrating care for people with co-occurring alcohol and other drug, medical, and mental health conditions. Alcohol Research \& Health, 33(4), 338- 349. Retrieved from http://pubs.niaaa.nih.gov/publications/arh334/338349.htm

Swahn, M., Haberlen, M., \& Palmier, J. (2014). Alcohol and drug use and other high-risk behaviors among youth in the slums of Kampala, Uganda: Perceptions and contexts obtained through focus groups. International Journal of Alcohol and Drug Research, 3(4), 289-295. doi: 10.7895/ijadr.v3i4.171

Swahn, M. H., Palmier, J. B., \& Kasirye, R. (2013). Alcohol exposures, alcohol marketing, and their associations with problem drinking and drunkenness among youth living in the slums of Kampala, Uganda. International Scholarly Research Notices Public Health, Article ID 948675, 9 pages. doi:10.1155/2013/948675

Ugandan Bureau of Statistics. (2014). National population and housing census: 2014 provisional results (revised edition). Kampala, Uganda: Ugandan Bureau of Statistics.

U.S. Agency for International Development. (2005). Survey of private health facilities in Uganda. Partners for Health Reformplus. Bethesda, MD, United States: Abt Associates Inc.

Willis, J. (2006). Drinking crisis? Change and continuity in cultures of drinking in Sub-Saharan Africa. African Journal of Drug \& Alcohol Studies, 5, 1-17.

World Bank. (2015). GDP per capita. Retrieved from http://data.worldbank.org/indicator/NY.GDP.PCAP.C $\mathrm{D}$

World Health Organization. (2010). Global strategy to reduce the harmful use of alcohol. Geneva, Switzerland: Author.

World Health Organization. (2014). WHO global status report on alcohol 2014 (Vol. 1). Geneva, Switzerland: WHO, Department of Mental Health and Substance Abuse. 